The Photopic Luminosity Curve and Visual Purple 505

\title{
SUMMARY
}

1. A case of bilateral tuberculous ulcer of the bulbar conjunctiva treated with streptomycin is described.

2. There was no response to penicillin therapy or lactic acid cauterisation.

3. The ulcers responded marvellously with streptomycin applied locally and parenterally.

4. There was no untoward complication after streptomycin therapy.

5. High concentrations of drops seem to have a better effect than low concentrations.

6. The ulcers seem to progress more favourably if the injections and drops are discontinued for some time after a few days of treatment.

7. After 7 days of treatment with streptomycin, scrapings from the floor of the ulcers failed to reveal the presence of any acidfast bacilli.

8. Sedimentation rate dropped from 40 to $10 \mathrm{~mm}$. per hour after streptomycin treatment.

Acknowledgment. We are very grateful to the Superintendent, Medical College Hospitals, Calcutta, for allowing us to publish this case. We are also indebted to Dr. P. K. Sarkar, Pathologist to the Eye Infirmary, Medical College Hospitals, Calcutta, who did the microscopic sections; to the artist of our institution, Mr. A. Das Gupta, who has kindly drawn the pictures; to Prof. B. P. Trivedi, for helping us to take the microphotograph; and to Dr. B. P. Neogy, of the Jadavpur Tuberculosis Hospital for valuable suggestions during the treatment of this case.

\section{THE PHOTOPIC LUMINOSITY CURVE AND VISUAL - PURPLE}

BY

L. C. THOMSON

From the Vision Research Unit, Medical Research Council, Institute of Ophthalmology, London:

RECENTLY Dartnall has suggested that the photopic as well as the scotopic luminosity may be mediated by the single pigment visual purple. He has deduced the shape and spectral position of the photopic luminosity curve of the eye by calculating the effect of an accumulation of indicator yellow upon the spectral distribution of the light absorbed by visual purple. He finds that the effect of increasing indicator yellow concentration is not, as might be 
expected, a steady displacement of the maximum of the curve towards red wavelengths, but that a limiting position is rapidly reached. The calculated luminosity curve corresponding to this limiting position agrees with the C.I.E. standard photopic curve.

Thus it would appear that the photorhemical activity upon which the sensation of photopic luminosity is based relates to one substance only, namely, visual purple. If this be true, however, we are forced to the unlikely conclusion that the mechanisms responsible for hue discrimination make no contribution to the overall sensitivity of the light-adapted eye. In other words Dartnall's hypothesis leaves no room for a luminosity contribution from any photo-sensitive substance other than visual purple, since the photopic sensitivity of the eye is, apparently, completely explained by supposing that it is mediated by a single substance, visual purple.

Thus the agreement between the derived curve and the photopic luminosity function, upon which the hypothesis basically relies for its verification, leads to the difficulty that the colour discrimination of the light-adapted eye cannot be easily explained.

On examination, however, the correspondence is seen to depend upon (a) the choice of the C.I.E. standard curve to represent the photopic sensitivity of the average eye, and (b) a correction applied to the data to allow for the absorption' of light by the yellow macular pigment.

(a) In 1924 Gibson and Tyndall measured the foveal photopic luminosity curve in 56 subjects and found that for wavelengths shorter than $500 \mu \mu$ their own mean curve showed departures, which were outside their experimental error, from the then existing American standard curve (I.E.S.). In recommending a new standard curve they did not, however, suggest any change in the region below $500 \mu \mu$, so that the present standard curve which is based upon Gibson and Tyndall's recommended figures still shows the departures from their mean results.

The extent of the departures may be seen in Fig. 1. Here log. luminosity (sensitivity) is plotted against the wavelength, and both Gibson and Tyndall's mean curve and the present C.I.E. standard curve are shown, when corrected by means of Ludvigh and McCarthy's data, to allow for the (pre-retinal) absorption of light in the optical system of the eye. The curves may therefore be regarded as a measure of the retinal, as distinct from the corneal, sensitivity. The data are for an equal quantum intensity spectrum. The curve marked $G$ and $T$ (C.F.S.) is for one of Gibson and Tyndall's more sensitive observers; that marked W.D.W., a sensitivity curve measured recently with the Wright colorimeter at Imperial College; both similarly corrected. 


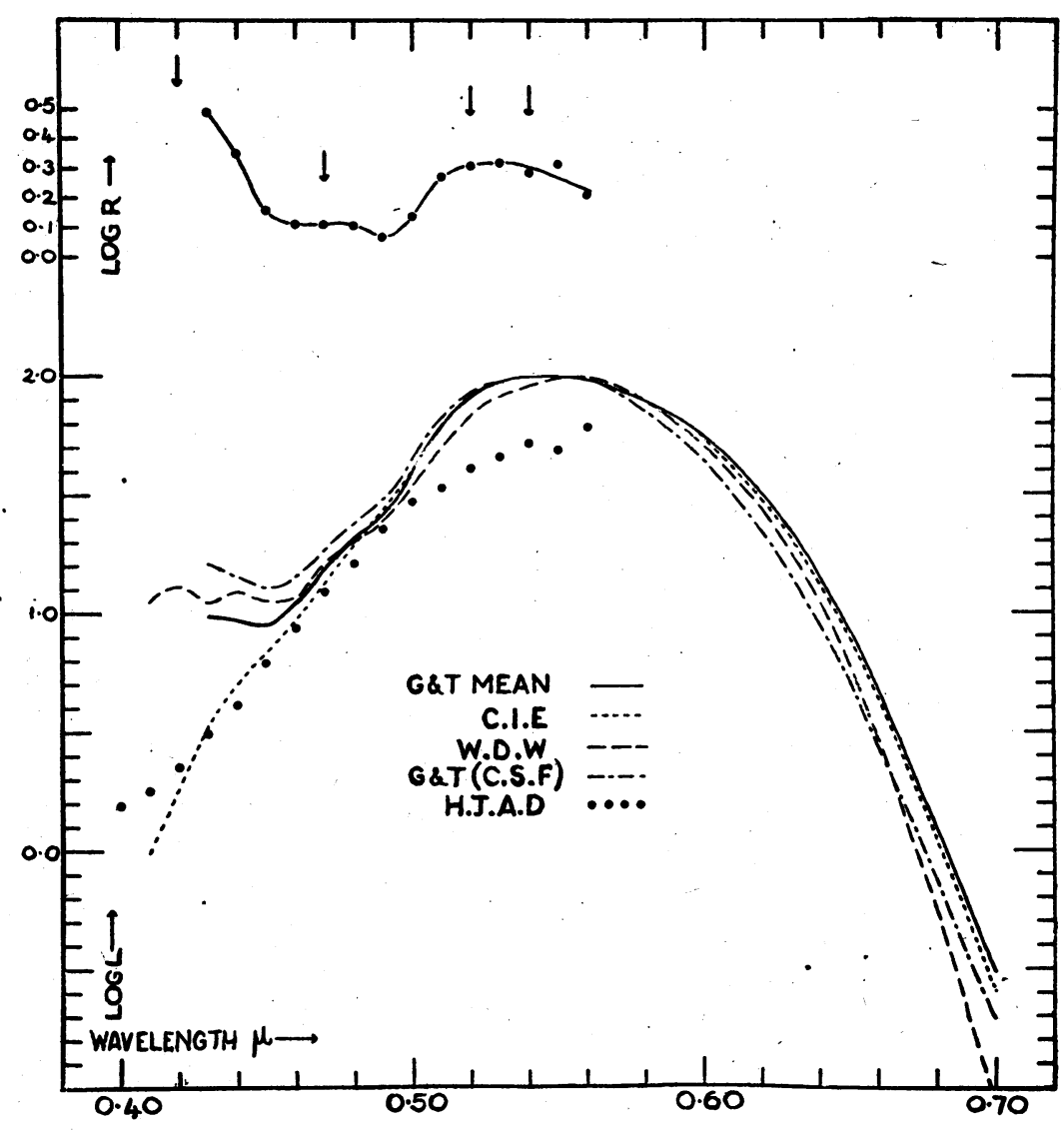

FIG. 1.

G \& T Mean ... Mean sensitivity of the eye as measured by Gibson and Tyndall.

C.I.E. ... ... The present standard photopic curve:

G \& T (C. F. S.) The sensitivity for one of Gibson and Tyndall's observers.

W.D.W. $\quad \ldots$ The sensitivity of one observer measured recently.

H.J.A.D. … Dartnall's derived curve, but without correction for macular absorption.

The arrows above the uppermost curve indicate the spectral regions in which modulator activity has been demorfstrated.

Thus the curve chosen by Dartnall, the C.I.E. curve, although " standard" in the sense that it is internationally agreed, does not, in fact, represent the average sensitivity of the eye below $500 \mu \mu$. 
(b) Polyak has stated that the yellow macular pigment lies in the ganglion and bipolar layers of the retina, and since the light does not traverse these layers to reach the foveal centre, and only to a small extent to reach a point displaced by $1^{\circ}$, it would seem that measurements of sensitivity made with a $2^{\circ}$ field (such as Gibson and Tyndall's), would be little affected by this pigmentation. Thus it would perhaps be more appropriate to omit a correction for macular pigment absorption. Dartnall's uncorrected ratios from Table I of his paper have, therefore, been plotted in Fig. 1. The set of points has been lowered to bring the point at $490 \mu \mu$ below Gibson and Tyndall's mean curve, and it can be seen that the agreement between the derived curve and the C.I.E. curve is in large measure dependent upon the macular pigment correction.

The factor by which the mean sensitivity of the eye (G. and T.) is greater than that derived by Dartnall is shown in the upper part of Fig. 1. This curve is a measure of the failure of the derived curve to describe the mean photopic sensitivity of the eye.

Are we then to discard Dartnall's hypothesis as incorrect? It may be, as he himself suggests, that the derived curve describes not the sensitivity of the light-adapted retina as a whole, but only the sensitivity of a separate brightness-perceiving mechanism playing no part in colour discrimination. This dissociation of luminosity and hue mechanisms is implicit in Granit's dominator-modulator theory of vision. The upper curve of Fig. 1 would then represent the contribution of the colour-perceiving mechanism to the sensitivity of the light-adapted eye. It is interesting to note that this curve does in fact show maxima of sensitivity in those very spectral regions in which Granit has found modulator activity to be most marked, these regions being shown in Fig. 1 by the arrows above the curve.

One merit of Dartnall's hypothesis is, therefore, that it enables an attempt to be made at separating the activity of the brightness mechanism from those of the colour-perceiving mechanisms in the human eye. Unfortunately, because of absence of data, the derived curve cannot at present be continued into the red region of the spectrum.

\section{REFERENCES}

- Dartalle, H. J. A. (1948).-Brit. J. Ophthal., 32, 793.

GIBSON, K. S. and TYNDALL, E. P. T. (1923-24).-Bur. Stand. Sci. Papers, 131. Granit, R. (1947).-Sensory Mechanisms of the Retina, Oxford Univ. Press. - (1948).-J. Neurophysiol. In press.

LUdvigh, E. and MCCARTHY, E. F. (1938).-Arch. Ophthal., $20,37$.

Polyax, S. L. (1941).-The Retina. Chicago: Univ. Chicago Press. 